\title{
Estudo comparativo das características históricas, sociodemográficas e de adesão dos usuários de substâncias psicoativas de um programa ambulatorial público
}

\author{
MARIA CECILIA FRICKE CHOHFI \\ Ambulatório de Especialidades Médicas (AME de Psiquiatria Dra. Jandira Masur), Universidade \\ Federal de São Paulo, São Paulo, SP, Brasil. \\ E-mail: cicachohfi@hotmail.com
}

\section{JULIANA DE ALMEIDA CASTRO MARINHO MEIRELLES}

Ambulatório de Especialidades Médicas (AME de Psiquiatria Dra. Jandira Masur), Universidade Federal de São Paulo, São Paulo, SP, Brasil.

E-mail: ju.deacmarinho@gmail.com

\section{CLAUDIO JERONIMO DA SILVA}

Ambulatório de Especialidades Médicas (AME de Psiquiatria Dra. Jandira Masur), Universidade Federal de São Paulo, São Paulo, SP, Brasil.

E-mail: claudio.jeronimo@uol.com.br

\section{Resumo}

Nos últimos anos, o consumo de substâncias lícitas e ilícitas no Brasil cresceu. Isso desencadeia problemas políticos, econômicos, sociais e culturais. Dessa forma, houve um aumento na demanda de tratamentos especializados. Nesse prisma, conhecer o perfil do usuário e a adesão ao tratamento é de suma importância para facilitar a gestão do serviço especializado. $\mathrm{O}$ objetivo deste estudo foi caracterizar a população atendida no programa de tratamento de álcool e drogas do Ambulatório Médico de Especialidades, comparando os dados do primeiro ano de funcionamento do serviço com os do segundo quanto às características históricas, sociodemográficas e de adesão ao tratamento dos pacientes. Trata-se de um estudo observacional, descritivo e retrospectivo. A amostra compreendeu 880 pacientes. Foi possível verificar associações entre as variáveis categóricas por meio dos testes qui-quadrado e exato de Fisher. Constatou-se a predominância do sexo 
masculino nos dois anos. No primeiro ano, solteiros buscaram tratamento e, no segundo, aumentou o número de casados. A idade média foi de 37 anos, com predomínio das classes sociais C e D no primeiro ano e E no segundo ano. A maioria realizou tratamento anterior, com média de 2,6 tratamentos; e 97,1\% fizeram uso de álcool, $85,8 \%$ de tabaco e $68,0 \%$, de maconha. A amostra do primeiro ano apresentou porcentagem maior de melhora $(8,0 \%$ contra $4,2 \%)$ e de abandono (39,2\% contra $26,5 \%)$. Quando se caracterizou o usuário, foi possível perceber, no perfil, fatos que corroboram o que outros pesquisadores já haviam identificado em usuários brasileiros (homens, de classes C, D e E, na faixa etária entre 30 e 40 anos); as drogas mais utilizadas são as lícitas, o que facilita o uso. Por ser uma doença recidivante, a média é de 2,6 tratamentos anteriores, o que afeta negativamente a adesão. Portanto, é importante que se conheçam esses dados para facilitar a gestão do tratamento, tornando-o mais eficaz.

\section{Palavras-chave}

Usuários de drogas. Assistência ambulatorial. Transtornos relacionados ao uso de substâncias. Administração de serviços de saúde. Registros médicos.

\section{INTRODUÇÃO}

Nos últimos anos, o índice de consumo de drogas lícitas e ilícitas cresceu e, sendo assim, tornou-se motivo de grande preocupação e debate em diversos países por desencadear problemas políticos, econômicos, sociais e culturais. Como defende o dr. Laranjeira, a dependência química é uma doença crônica, incurável e progressiva, ou seja, recidivante, e o uso continuado provoca mudanças na estrutura e no funcionamento do cérebro (LARANJEIRA; RIBEIRO, 2010).

Além disso, existem vários outros problemas sociais associados ao uso de substâncias e à dependência química, como aumento da violência doméstica, mortes no trânsito, narcotráfico, roubos, assassinatos, falta de tratamentos especializados, familiares desesperados, corrupção, dificuldades dos tratamentos ambulatoriais e muitas outras questões relacionadas (CORDEIRO; DIEHL; MARI, 2019).

De acordo com o II Levantamento Nacional de Álcool e Drogas (LARANJEIRA, 2014), o mais recente relatório realizado pelo Instituto Nacional de Ciência e Tecnologia para Políticas Públicas do Álcool e outras Drogas (Inpad), não houve um aumento do número de pessoas que consomem álcool no Brasil, 
mas as que já utilizavam a substância passaram a fazê-lo com maior frequência. As mulheres (e especialmente as mais jovens) são a população mais vulnerável, apresentando maiores índices de aumento entre 2006 e 2012 e bebendo de forma mais nociva. No Brasil, pelo menos $7 \%$ da população adulta já experimentou maconha na vida e $3 \%$ usam maconha de forma frequente, totalizando mais de 3 milhões de pessoas. Adultos usam o mesmo que adolescentes, e, no último ano, a prevalência de uso das diversas apresentações de cocaína atingiu 2,6 milhões de adultos e 244 mil adolescentes.

Apesar das estratégias do Ministério da Saúde, sabe-se que a dependência química causa um grande impacto social e econômico, com altos custos anuais para o tratamento dos dependentes. Entretanto, observa-se que as políticas públicas vêm caminhando de forma desproporcional à crescente velocidade dessa realidade e que, apesar de destinarem grande parte do orçamento público para essa área, ainda deparam com a precariedade e a falta de serviços especializados (DIEHL; SANTANA, 2019).

Com todo um histórico carente de serviços especializados, combinado a um fenômeno crescente, a dependência química vem recebendo foco nos últimos anos. Dessa forma, busca-se atualmente organizar um serviço de tratamento de excelência, criando-se um paradigma para profissionais e gestores de saúde que atuem pautados em uma organização empírica a partir do empenho e da experiência pessoal de profissionais capacitados (CORDEIRO; DIEHL; LARANJEIRA, 2019).

Conforme Laranjeira e Ribeiro (2010), a organização do sistema de tratamento da dependência química está repleta de problemas estruturais e organizacionais que afetam, de forma negativa, a efetividade do serviço prestado. Porém, nas últimas décadas, houve um aumento no interesse de pesquisadores sobre como é realizada a prestação de serviços nessa área, tanto pública quanto privada, e houve também uma produção científica embrionária sobre o assunto.

Apesar dos serviços substitutivos com o intuito de implementar novos dispositivos (mais eficazes no sentido de não produzirem novas formas de enclausuramento e hegemonias), os serviços têm sido considerados insuficientes e têm apresentado dificuldades em fornecer aos pacientes um atendimento eficiente que os ajude a viver de forma satisfatória na comunidade (CHEDA et al., 2010).

Um modelo pautado na intervenção breve, estruturado e de tempo limitado vem sendo considerado como uma alternativa eficiente de serviço. Obser- 
vam-se resultados terapêuticos positivos com minimização de custos e, ainda, evidências de eficácia no tratamento e na prevenção de problemas ligados ao consumo excessivo de drogas. Técnicas de entrevista motivacional e cognitivo-comportamental, escalas de avaliação e material de mútua ajuda vêm sendo utilizados e testados na rede primária de saúde e podem ser adotados por qualquer profissional capacitado, como médicos ou enfermeiros (SILVA, 2013).

O tratamento ambulatorial da dependência de substâncias psicoativas agrega vantagens como: custo inferior às internações hospitalares, atendimento a um número maior de pacientes e permanência deles por mais tempo no serviço, possibilitando uma participação maior no programa (ZANELATTO, 2019).

O Ambulatório Médico de Especialidades (AME) de Psiquiatria nasceu com a proposta inovadora de atenção integral à saúde mental com várias especialidades de tratamento, sendo uma delas em álcool e drogas. Resultado de uma parceria entre o governo do estado de São Paulo, os departamentos de psiquiatria das faculdades de Medicina do município de São Paulo - Universidade Federal de São Paulo (Unifesp), Universidade de São Paulo (USP), Faculdade de Ciências Médicas da Santa Casa de São Paulo (FCMSCSP), Universidade Santo Amaro (Unisa) -, o Conselho Regional de Medicina do Estado de São Paulo e o Ministério Público Estadual, o AME de Psiquiatria se constituiu em uma proposta de tratamento breve. Mesmo com tamanho esforço do governo nesse sentido, ainda é grande o número de pacientes que desistem antes do término do tratamento. Tal ocorrência é altamente prejudicial às políticas direcionadas aos usuários de álcool e outras drogas, uma vez que a eficácia de qualquer tratamento depende estritamente da adesão do paciente (CHEDA et al., 2010).

O AME de Psiquiatria trabalha com enfoque no tratamento personalizado (o Plano Individual de Atendimento - PIA) e na busca ativa, ou seja, o serviço apresenta um diferencial no que diz respeito à conduta com o paciente, o que corrobora que, ao ser contatado e monitorado periodicamente, o usuário permanece mais tempo em tratamento. Um dos fatores geradores é o apoio motivacional dado pela equipe: o paciente não deve se perder em seu acompanhamento, e, caso isso ocorra, esforça-se para que ele volte. Nesse prisma, o processo utilizado também se baseia na participação dos familiares dos pacientes, que são convidados a integrar o tratamento de maneira cooperativa.

Ainda na mesma linha de raciocínio, Aslani et al. (2009) afirmam que a não adesão é um fenômeno que causa um grande problema para o tratamen- 
to, principalmente se existe doença crônica combinada. No mesmo diapasão, seguem Leite e Vasconcellos (2003), ao afirmarem que o sucesso do tratamento (tanto da terapia como do controle e da prevenção de qualquer patologia) depende de sua adesão. Uma sugestão para os desafios encontrados no Brasil não seria a oferta desses tratamentos, mas a adequação às abordagens propostas, visando a uma maior adesão. Por tudo isto, acredita-se na essencialidade do estudo das características dessa população a fim de possibilitar a melhor compreensão do fenômeno em questão.

O objetivo deste estudo, além de conhecer o funcionamento do tratamento ambulatorial, foi comparar o perfil sociodemográfico do paciente, suas características históricas e sua adesão, ou seja, o tempo que ele permaneceu, combinado ao tipo de alta. Por características históricas, entende-se qualquer processo relacionado ao histórico de uso da substância pelo paciente (por exemplo: quantidade de tratamentos anteriores, expectativa em relação ao tratamento ou substâncias preferidas). Acredita-se que conhecer a população específica usuária do tratamento oferecido poderá facilitar a gestão do serviço especializado em dependência química, auxiliando na elaboração de estratégias de prevenção e tratamento e buscando integrar profissionais da saúde, familiares e sociedade.

\section{METODOLOGIA}

\section{Local e amostra}

O estudo foi conduzido no AME de Psiquiatria Dra. Jandira Masur, localizado na cidade de São Paulo, na microrregião da Vila Maria/Vila Guilherme. A amostra foi colhida de todos os prontuários dos pacientes que frequentaram o serviço nos dois primeiros anos de funcionamento. Consideraram-se, no estudo, 425 pacientes que iniciaram o tratamento no primeiro ano e 455 no segundo.

\section{Procedimentos utilizados}

Trata-se de um estudo observacional e descritivo dos dados colhidos dos prontuários supracitados. É importante frisar que nem todas as questões do prontuário foram respondidas por todos os pacientes, mas a amostra conseguida já é suficientemente densa para a análise deste estudo. 


\section{Análise estatística}

Os dados foram categorizados e apresentados em frequências absolutas e relativas. Para as variáveis numéricas, apresentaram-se medidas-resumo (média, mínimo, máximo, quartis e desvio padrão). A existência de associações entre variáveis categóricas foi verificada por meio do teste qui-quadrado ou exato de Fisher (em caso de amostras pequenas). As comparações de médias entre dois grupos e mais de dois grupos foram realizadas pelos testes $t$ de Student e análise de variância, respectivamente. Ambos os testes têm como pressuposto a normalidade dos dados, o que foi verificado utilizando-se o teste de Kolmogorov-Smirnov. Para todos os testes estatísticos, adotou-se um nível de significância de 5\%. Para amostras independentes, utilizou-se somente $\mathrm{o}$ teste $\mathrm{t}$ de Student.

\section{Aspectos éticos}

Em relação à questão ética, como as informações foram coletadas de prontuários clínicos, não há a necessidade do Termo de Consentimento Livre e Esclarecido, conforme preconizam os documentos internacionais e a Resolução n. 196/1996 do Ministério da Saúde.

\section{RESULTADOS}

A amostra estudada apresenta, na Tabela 1, a distribuição dos pacientes por características sociodemográficas. De acordo com essa tabela, observam-se distribuições distintas entre o primeiro ano de funcionamento do serviço e o segundo em relação ao estado civil $(p=0,030)$, escolaridade $(p=0,022)$ e classe social ( $p=0,019)$. Para as demais variáveis, as distribuições apresentaram-se similares entre os dois períodos.

Denota-se uma porcentagem maior de solteiros no primeiro ano $(45,7 \%$ contra $37,1 \%$ do segundo ano), enquanto, no segundo ano, verificou-se uma maior porcentagem de separados $(21,2 \%$ contra $13,1 \%)$. Com relação à escolaridade, observou-se, no primeiro ano, uma porcentagem maior de alfabetizados $(2,1 \%$ contra $0,3 \%)$ e de ensino médio completo $(30,7 \%$, contra $22,1 \%)$. Verifica-se, ainda no segundo ano, presença de pacientes da classe E $(4,1 \%)$, grupo ausente no primeiro ano. A média de idade foi de 37,2 anos, com médias semelhantes por período. 
Tabela 1 Distribuição dos pacientes por características sociodemográficas

\begin{tabular}{|c|c|c|c|c|c|c|}
\hline & \multicolumn{4}{|c|}{ Período } & \multirow{2}{*}{\multicolumn{2}{|c|}{ Total }} \\
\hline & \multicolumn{2}{|c|}{ Primeiro ano } & \multicolumn{2}{|c|}{ Segundo ano } & & \\
\hline & $\mathbf{N}$ & $\%$ & $\mathbf{N}$ & $\%$ & $\mathbf{N}$ & $\%$ \\
\hline Gênero & 424 & $100,0 \%$ & 449 & $100,0 \%$ & 873 & $100,0 \%$ \\
\hline Feminino & 83 & $19,6 \%$ & 77 & $17,1 \%$ & 160 & $18,3 \%$ \\
\hline Masculino & 341 & $80,4 \%$ & 372 & $82,9 \%$ & 713 & $81,7 \%$ \\
\hline \multicolumn{7}{|c|}{$\operatorname{Chi}(1)=0,86(p=0,354)$} \\
\hline Faixa etária & 415 & $100,0 \%$ & 399 & $100,0 \%$ & 814 & $100,0 \%$ \\
\hline Até 20 anos & 29 & $7,0 \%$ & 33 & $8,3 \%$ & 62 & $7,6 \%$ \\
\hline De 20 a 30 anos & 108 & $26,0 \%$ & 104 & $26,1 \%$ & 212 & $26,0 \%$ \\
\hline De 30 a 40 anos & 122 & $29,4 \%$ & 112 & $28,1 \%$ & 234 & $28,7 \%$ \\
\hline De 40 a 50 anos & 87 & $21,0 \%$ & 85 & $21,3 \%$ & 172 & $21,1 \%$ \\
\hline Acima de 50 anos & 69 & $16,6 \%$ & 65 & $16,3 \%$ & 134 & $16,5 \%$ \\
\hline \multicolumn{7}{|c|}{$\operatorname{Chi}(4)=0,59(p=0,964)$} \\
\hline Estado civil & 337 & $100,0 \%$ & 345 & $100,0 \%$ & 682 & $100,0 \%$ \\
\hline Solteiros & 154 & $45,7 \%$ & 128 & $37,1 \%$ & 282 & $41,3 \%$ \\
\hline Casados & 85 & $25,2 \%$ & 91 & $26,4 \%$ & 176 & $25,8 \%$ \\
\hline Em união estável & 48 & $14,2 \%$ & 50 & $14,5 \%$ & 98 & $14,4 \%$ \\
\hline Separados & 44 & $13,1 \%$ & 73 & $21,2 \%$ & 117 & $17,2 \%$ \\
\hline Viúvos & 6 & $1,8 \%$ & 3 & $0,9 \%$ & 9 & $1,3 \%$ \\
\hline \multicolumn{7}{|c|}{$\operatorname{Chi}(4)=10,74(p=0,030)$} \\
\hline Escolaridade & 332 & $100,0 \%$ & 339 & $100,0 \%$ & 671 & $100,0 \%$ \\
\hline Analfabeto & 9 & $2,7 \%$ & 11 & $3,2 \%$ & 20 & $3,0 \%$ \\
\hline Alfabetizado & 7 & $2,1 \%$ & 1 & $0,3 \%$ & 8 & $1,2 \%$ \\
\hline
\end{tabular}




\begin{tabular}{|c|c|c|c|c|c|c|}
\hline & \multicolumn{4}{|c|}{ Período } & \multirow{2}{*}{\multicolumn{2}{|c|}{ Total }} \\
\hline & \multicolumn{2}{|c|}{ Primeiro ano } & \multicolumn{2}{|c|}{ Segundo ano } & & \\
\hline & $\mathbf{N}$ & $\%$ & $\mathbf{N}$ & $\%$ & $\mathbf{N}$ & $\%$ \\
\hline Fundamental incompleto & 95 & $28,6 \%$ & 100 & $29,5 \%$ & 195 & $29,1 \%$ \\
\hline Fundamental completo & 39 & $11,7 \%$ & 41 & $12,1 \%$ & 80 & $11,9 \%$ \\
\hline Médio incompleto & 47 & $14,2 \%$ & 64 & $18,9 \%$ & 111 & $16,5 \%$ \\
\hline Médio completo & 102 & $30,7 \%$ & 75 & $22,1 \%$ & 177 & $26,4 \%$ \\
\hline Superior incompleto & 22 & $6,6 \%$ & 29 & $8,6 \%$ & 51 & $7,6 \%$ \\
\hline Superior completo & 9 & $2,7 \%$ & 18 & $5,3 \%$ & 27 & $4,0 \%$ \\
\hline Pós-graduado & 2 & $0,6 \%$ & 0 & $0 \%$ & 2 & $0,3 \%$ \\
\hline \multicolumn{7}{|c|}{ Teste exato de Fisher $(p=0,022)$} \\
\hline Classe social & 184 & $100,0 \%$ & 123 & $100,0 \%$ & 307 & $100,0 \%$ \\
\hline$A 2$ & 1 & $0,5 \%$ & 2 & $1,6 \%$ & 3 & $1,0 \%$ \\
\hline B1 & 14 & $7,6 \%$ & 3 & $2,4 \%$ & 17 & $5,5 \%$ \\
\hline B2 & 28 & $15,2 \%$ & 21 & $17,1 \%$ & 49 & $16,0 \%$ \\
\hline C & 99 & $53,8 \%$ & 60 & $48,8 \%$ & 159 & $51,8 \%$ \\
\hline D & 42 & $22,8 \%$ & 32 & $26,0 \%$ & 74 & $24,1 \%$ \\
\hline E & 0 & $0 \%$ & 5 & $4,1 \%$ & 5 & $1,6 \%$ \\
\hline Teste exato de Fisher $(p=c$ & & & & & & \\
\hline
\end{tabular}

Fonte: Elaborada pelos autores.

Conforme a Tabela 2, nota-se que, exceto para inalantes e alucinógenos (para os quais ocorreu um aumento no segundo período), os históricos de uso de substâncias foram similares entre os períodos. Observa-se que, de uma forma geral, 97,1\% fizeram uso de álcool, 85,8\% de tabaco e 68,0\% de maconha. 
Tabela 2 Distribuição dos pacientes por histórico de uso de substâncias' (afirmativas)

\begin{tabular}{lcccccccc}
\hline \multirow{2}{*}{$\begin{array}{l}\text { Histórico de uso } \\
\text { de substância }\end{array}$} & \multicolumn{9}{c}{ Primeiro ano } & \multicolumn{2}{c}{ Segundo ano } & & \multicolumn{2}{c}{ Total } & p \\
\cline { 2 - 5 } & N & N & N & \% & N & \% & \\
\hline Álcool & 272 & $97,1 \%$ & 255 & $97,0 \%$ & 527 & $97,1 \%$ & 0,899 \\
\hline Tabaco & 237 & $85,9 \%$ & 221 & $85,7 \%$ & 458 & $85,8 \%$ & 0,944 \\
\hline Maconha & 186 & $66,9 \%$ & 178 & $69,3 \%$ & 364 & $68,0 \%$ & 0,560 \\
\hline Cocaína & 179 & $64,2 \%$ & 172 & $66,9 \%$ & 351 & $65,5 \%$ & 0,501 \\
\hline Inalantes & 84 & $30,4 \%$ & 104 & $42,3 \%$ & 188 & $36,0 \%$ & 0,005 \\
\hline Crack & 96 & $34,8 \%$ & 86 & $34,3 \%$ & 182 & $34,5 \%$ & 0,900 \\
\hline Ecstasy & 37 & $13,5 \%$ & 47 & $19,5 \%$ & 84 & $16,3 \%$ & 0,066 \\
\hline Alucinógenos & 29 & $10,7 \%$ & 40 & $16,8 \%$ & 69 & $13,5 \%$ & 0,043 \\
\hline Hipnóticos & 32 & $11,8 \%$ & 22 & $9,3 \%$ & 54 & $10,6 \%$ & 0,357 \\
\hline Outras & 31 & $11,7 \%$ & 20 & $9,0 \%$ & 51 & $10,5 \%$ & 0,334 \\
\hline Anfetamina & 19 & $6,9 \%$ & 25 & $10,5 \%$ & 44 & $8,6 \%$ & 0,151 \\
\hline
\end{tabular}

${ }^{1}$ Resposta de múltipla escolha; $\mathrm{p}$ : nível descritivo do teste de qui-quadrado.

Fonte: Elaborada pelos autores.

Na Tabela 3, é possível observar que não houve diferença entre as médias distintas de número de tratamentos anteriores $(\mathrm{p}=0,744)$. Assim, de forma geral, os pacientes que realizaram algum tratamento anterior fizeram, em média, 2,6 tratamentos ( $\mathrm{DP}=2,5$ tratamentos). Isso, em verdade, é um dado comum de estudos em dependência química - geralmente, são várias as tentativas de tratamentos anteriores.

Tabela 3 Medidas-resumo do número de tentativas de tratamento realizadas anteriormente

\begin{tabular}{lcccccccc}
\hline & Média & $\begin{array}{c}\text { Desvio } \\
\text { padrão }\end{array}$ & Mín. & Máx. & $\begin{array}{c}\mathbf{1 0} \\
\text { Quartil }\end{array}$ & Mediana & $\begin{array}{c}\text { 3o } \\
\text { Quartil }\end{array}$ & N \\
\hline Total & $\mathbf{2 , 6}$ & $\mathbf{2 , 5}$ & $\mathbf{1 , 0}$ & $\mathbf{2 0 , 0}$ & $\mathbf{1 , 0}$ & $\mathbf{2 , 0}$ & $\mathbf{3 , 0}$ & $\mathbf{2 8 1}$ \\
\hline Primeiro ano & 2,6 & 2,2 & 1,0 & 11,0 & 1,0 & 2,0 & 3,0 & 158 \\
\hline Segundo ano & 2,7 & 2,8 & 1,0 & 20,0 & 1,0 & 2,0 & 3,0 & 123 \\
\hline$t=0,33(p=0,744)$ & & & & & & & \\
\hline
\end{tabular}

Fonte: Elaborada pelos autores. 
A Tabela 4 mostra que as taxas de adesão dos pacientes ao tratamento não foram similares entre os períodos ( $\mathrm{p}<0,001)$. Nota-se, no segundo ano, uma porcentagem maior de pacientes em tratamento $(53,7 \%$ contra $35,0 \%$ do primeiro ano). O primeiro ano apresentou porcentagem maior de melhora ( $8,0 \%$ contra $4,2 \%$ do segundo ano) e abandono (39,2\% contra $26,5 \%)$.

Tabela 4 Distribuição dos pacientes por status no tratamento

\begin{tabular}{|c|c|c|c|c|c|c|}
\hline \multirow{3}{*}{ Situação do paciente } & \multicolumn{4}{|c|}{ Período } & \multirow{2}{*}{\multicolumn{2}{|c|}{ Total }} \\
\hline & \multicolumn{2}{|c|}{ Primeiro ano } & \multicolumn{2}{|c|}{ Segundo ano } & & \\
\hline & $\mathbf{N}$ & $\%$ & $\mathbf{N}$ & $\%$ & $\mathbf{N}$ & $\%$ \\
\hline TOTAL & 423 & $100,0 \%$ & 449 & $100,0 \%$ & 872 & $100,0 \%$ \\
\hline Abandono & 166 & $39,2 \%$ & 119 & $26,5 \%$ & 285 & $32,7 \%$ \\
\hline Abandono precoce & 46 & $10,9 \%$ & 52 & $11,6 \%$ & 98 & $11,2 \%$ \\
\hline Melhora & 34 & $8,0 \%$ & 19 & $4,2 \%$ & 53 & $6,1 \%$ \\
\hline Encaminhamento & 27 & $6,4 \%$ & 16 & $3,6 \%$ & 43 & $4,9 \%$ \\
\hline Pedida & 2 & $0,5 \%$ & 2 & $0,4 \%$ & 4 & $0,5 \%$ \\
\hline Em tratamento & 148 & $35,0 \%$ & 241 & $53,7 \%$ & 389 & $44,6 \%$ \\
\hline $\operatorname{Chi}(5)=36,67(p<0,001$ & & & & & & \\
\hline
\end{tabular}

Fonte: Elaborada pelos autores.

A Tabela 5 apresenta distribuições distintas de status do paciente no tratamento por diagnóstico somente no primeiro período $(\mathrm{p}=0,005)$. Assim, nota-se uma porcentagem maior de abandono no grupo de pacientes com mais de três diagnósticos $(85,7 \%)$, comparativamente aos demais grupos de diagnóstico. Na mesma tabela, aparece maior porcentagem de abandono precoce no grupo de pacientes diagnosticados somente como F10 (F10.1 ou F10.2). Destaca-se ainda que o grupo de pacientes diagnosticados como usuários somente de crack apresentou maior porcentagem de melhora em relação aos demais grupos $(41,7 \%)$. 
Tabela 5 Distribuição dos pacientes por status no tratamento (abandono, abandono precoce e melhora)

\begin{tabular}{|c|c|c|c|c|c|c|c|c|}
\hline & \multicolumn{6}{|c|}{ Status do paciente } & \multirow{2}{*}{\multicolumn{2}{|c|}{ Total }} \\
\hline & \multicolumn{2}{|c|}{ Abandono } & \multicolumn{2}{|c|}{$\begin{array}{l}\text { Abandono } \\
\text { precoce }\end{array}$} & \multicolumn{2}{|c|}{ Melhora } & & \\
\hline & $\mathbf{N}$ & $\%$ & $\mathbf{N}$ & $\%$ & $\mathbf{N}$ & $\%$ & $\mathbf{N}$ & $\%$ \\
\hline Primeiro ano & 145 & $70,0 \%$ & 30 & $14,5 \%$ & 32 & $15,5 \%$ & 207 & $100,0 \%$ \\
\hline Somente F10 & 35 & $63,6 \%$ & 13 & $23,6 \%$ & 7 & $12,7 \%$ & 55 & $100,0 \%$ \\
\hline Somente F12 & 3 & $60,0 \%$ & 1 & $20,0 \%$ & 1 & $20,0 \%$ & 5 & $100,0 \%$ \\
\hline Somente F13 & 0 & $0,0 \%$ & 2 & $100,0 \%$ & 0 & $0,0 \%$ & 2 & $100,0 \%$ \\
\hline Somente F14 & 3 & $75,0 \%$ & 1 & $25,0 \%$ & 0 & $0,0 \%$ & 4 & $100,0 \%$ \\
\hline Somente F17 & 2 & $66,7 \%$ & 0 & $0,0 \%$ & 1 & $33,3 \%$ & 3 & $100,0 \%$ \\
\hline Somente crack & 5 & $41,7 \%$ & 2 & $16,7 \%$ & 5 & $41,7 \%$ & 12 & $100,0 \%$ \\
\hline Dois diagnósticos & 43 & $68,3 \%$ & 7 & $11,1 \%$ & 13 & $20,6 \%$ & 63 & $100,0 \%$ \\
\hline $\begin{array}{l}\text { Três ou mais } \\
\text { diagnósticos }\end{array}$ & 54 & $85,7 \%$ & 4 & $6,3 \%$ & 5 & $7,9 \%$ & 63 & $100,0 \%$ \\
\hline \multicolumn{9}{|c|}{ Teste exato de Fisher $(p=0,005)$} \\
\hline Segundo ano & 92 & $76,7 \%$ & 13 & $10,8 \%$ & 15 & $12,5 \%$ & 120 & $100,0 \%$ \\
\hline Somente F10 & 22 & $66,7 \%$ & 5 & $15,2 \%$ & 6 & $18,2 \%$ & 33 & $100,0 \%$ \\
\hline Somente F12 & 2 & $66,7 \%$ & 0 & $0,0 \%$ & 1 & $33,3 \%$ & 3 & $100,0 \%$ \\
\hline Somente F14 & 4 & $66,7 \%$ & 1 & $16,7 \%$ & 1 & $16,7 \%$ & 6 & $100,0 \%$ \\
\hline Somente F17 & 1 & $100,0 \%$ & 0 & $0,0 \%$ & 0 & $0,0 \%$ & 1 & $100,0 \%$ \\
\hline Somente crack & 3 & $100,0 \%$ & 0 & $0,0 \%$ & 0 & $0,0 \%$ & 3 & $100,0 \%$ \\
\hline Dois diagnósticos & 23 & $76,7 \%$ & 3 & $10,0 \%$ & 4 & $13,3 \%$ & 30 & $100,0 \%$ \\
\hline $\begin{array}{l}\text { Três ou mais } \\
\text { diagnósticos }\end{array}$ & 37 & $84,1 \%$ & 4 & $9,1 \%$ & 3 & $6,8 \%$ & 44 & $100,0 \%$ \\
\hline
\end{tabular}

Fonte: Elaborada pelos autores. 


\section{DISCUSSÃO}

A análise quanto ao perfil sociodemográfico demonstrou o que comumente vem sido apresentado nos levantamentos epidemiológicos do uso de substâncias pelos brasileiros, a saber: homem, desempregado, classes C e D, faixa etária entre 30 e 40 anos. Esses resultados estão de acordo com vários outros estudos (LARANJEIRA, 2014; CAPISTRANO et al., 2013; LARANJEIRA; RIBEIRO, 2010; BELL et al., 2009; CHEDA et al., 2010; ARRUDA et al., 2019), os quais também descrevem o perfil de dependentes químicos com as mesmas características.

Ainda nesse diapasão, corroboram Garcia, Siqueira e Souza (2005), que, em sua amostra estudada, a prevalência de pacientes era do sexo masculino em ambos os grupos $(89,8 \%$ contra $82,1 \% ; p=0,072)$. O estudo foi feito com amostra de 216 pacientes do Programa de Atendimento ao Alcoolista do Hospital Universitário Cassiano Antônio Moraes, da Universidade Federal do Espírito Santo, com média de $88,5 \%$ de pacientes do sexo masculino. Um estudo mais atual realizado por Arruda et al. (2019) no Acre também identificou um perfil quase idêntico ao deste trabalho, com predominância de 87,1\% de usuários do sexo masculino, na faixa etária entre 36 e 45 anos.

De acordo com Capistrano et al. (2013, p. 238),

[...] pode-se inferir que as abordagens terapêuticas utilizadas no tratamento do
dependente químico, em um hospital psiquiátrico ou nos serviços substitutivos,
como os Centros de Atenção Psicossocial Álcool e Drogas (CAPSad), são mais
eficazes em pacientes com idades mais avançadas, que utilizam drogas há mui-
to tempo e, em decorrência do uso contínuo, já tiveram um intenso sofrimento.

Nesse sentido, saber a idade do usuário por meio de seu perfil pode influir positivamente na melhor escolha para seu tratamento.

Neste estudo, ainda se verificou que a maioria dos usuários analisados nos dois anos (29,5\% no primeiro ano e $29,1 \%$ no segundo) possuem ensino fundamental incompleto. Almeida et al. (2014) também desenvolveram uma pesquisa e identificaram dados similares. Segundo Pechansky, Szobot e Scivoletto (2004), vários danos cognitivos são causados pelo excesso de uso de substâncias psicoativas, o que acaba dificultando o processo de aprendizagem e gerando problemas de memorização. Por sua vez, isso pode incidir em abandono escolar - o que justifica, dessa forma, o baixo nível de escolaridade desses usuários. De acordo ainda com os autores: 
O consumo de álcool na adolescência também está associado a uma série de prejuízos acadêmicos. Esses podem decorrer do déficit de memória: adolescentes com dependência de álcool apresentam mais dificuldade em recordar palavras e desenhos geométricos simples após um intervalo de 10 minutos, em comparação a adolescentes sem dependência alcoólica. Sabendo-se que a memória é função fundamental no processo de aprendizagem e que esta se altera com o consumo de álcool, é natural que este também comprometa o processo de aprendizagem (PECHANSKY; SZOBOT; SCIVOLETTO, 2004, p. 16).

O álcool foi identificado como sendo a substância psicoativa mais utilizada pelos usuários nos dois períodos verificados, com números extremamente próximos (97,1\% e 97\%, respectivamente). Em vários outros estudos, como os de Silva, Guimarães e Salles (2014) e Ribeiro e Carvalho (2015), a porcentagem de usuários de álcool é superior a 70\%. Ainda de acordo com Silva, Guimarães e Salles (2014, p. 1011):

Álcool foi a substância psicoativa de maior consumo (72,0\%) de prevalência. Acredita-se que um dos fatores que corrobora para o consumo elevado desta substância é o fato de ser lícita e, portanto, de fácil acesso.

A média de tratamentos anteriores na amostra dos dois anos foi de 2,6. No segundo ano, aumentou-se a busca por tratamento pela "primeira vez", o que é comum a dependentes químicos, universo em que, geralmente, são várias as tentativas de tratamentos anteriores (CHAVES et al., 2011; ARAÚJO et al., 2008; NAPPO; OLIVEIRA, 2008).

Além de a busca por tratamento ter, em sua maioria, o motivo familiar, um dos dados que se destacaram no estudo foi o da porcentagem de casados ter aumentado no segundo ano. A importância da família e de seus vínculos como base para a reestruturação da vida dos dependentes serve como fator motivador na busca pela reabilitação.

Quanto aos diagnósticos, não houve mudanças entre os dois períodos, e, conforme estudo realizado, esse dado reforça a descrição do perfil do brasileiro usuário de substâncias entorpecentes (LARANJEIRA, 2014). Contudo, ressalta-se o dado de que houve uma maior porcentagem de abandono precoce no grupo de pacientes diagnosticados somente como F10 (F10.1 ou F10.2, ou seja, transtornos mentais e comportamentais devidos ao uso de álcool), e essa causa poderia ser investigada em estudos posteriores. 
De acordo com Figlie e Laranjeira (2004), a ação de oferecer ao paciente várias chances para se tratar é uma oportunidade e tem o papel facilitador para a adesão - procedimento que é realizado no AME ao oferecer o serviço de busca ativa ao paciente por meio de ligações. $\mathrm{O}$ intuito desse procedimento é mostrar atenção e, ao mesmo tempo, dar uma nova chance.

Refletir sobre a importância da abordagem motivacional na rede de saúde desde o momento do encaminhamento ao serviço especializado (assim como o estabelecimento de um bom vínculo e monitoramento por meio de gerenciamento de caso) pode facilitar a adesão dessa população específica. Novos estudos poderão ser realizados com esse objetivo. Destaca-se ainda que o grupo de pacientes diagnosticados como usuários somente de crack apresentou maior porcentagem de melhora em relação aos demais grupos $(41,7 \%)$.

O objetivo do AME, desde a sua criação, é otimizar o atendimento (principalmente aos pacientes mentais mais graves) para complementar e fortalecer o atual sistema de rede com foco na saúde mental, como Centro de Atenção Psicossocial (Caps), unidade básica de saúde (UBS), Centro de Convivência e Cooperativa (Cecco) e Núcleo de Apoio à Saúde da Família (Nasf) (LARANJEIRA, 2014).

A importância de se conhecer o perfil sociodemográfico do paciente é um dos fatores intrínsecos para se analisar a forma mais eficaz de se aderir ao programa oferecido, e o AME desenvolve uma combinação de terapias e trabalhos em rede com discussões periódicas dos casos e com uma equipe treinada e direcionada para as necessidades dos pacientes (FERREIRA; MARINHO; SILVA, 2019).

\section{CONCLUSÃO}

Conhecer o tratamento especializado e seu funcionamento possibilitou, por meio de pesquisa de prontuários, traçar o perfil do paciente que, de acordo com os dados, corrobora o perfil do brasileiro (homem, desempregado, classes C e D, faixa etária entre 30 e 40 anos) no que diz respeito ao uso de substâncias psicoativas, sendo as mais consumidas o álcool (97\% e 97,1\%), o tabaco $(85,9 \%$ e $85,7 \%)$ e a maconha $(66,9 \%$ e $69,3 \%)$ no primeiro e no segundo ano (dados respectivos).

A existência de espaços de apoio e orientação (assim como o encaminhamento de familiares à rede de saúde básica e a grupos de mútua ajuda quando necessário) parece ajudar na busca do paciente que deseja se tratar e na sua 
adesão ao programa. O AME de Psiquiatria trabalha com enfoque no tratamento personalizado e na busca ativa, ou seja, esse serviço é um diferencial no trato com o paciente. Dessa forma, confirma-se que, ao ser contatado e monitorado periodicamente, o usuário permanece mais tempo em tratamento (o que reflete também nas taxas de abandono, que diminuíram na comparação entre os dois anos - $39,2 \%$ e $26,5 \%$, respectivamente), e um dos fatores geradores disso é o apoio motivacional dado pela equipe que está sempre atenta para que o paciente não se perca no acompanhamento realizado; no entanto, se isso ocorrer, esforços serão realizados para que ele retorne ao programa.

Assim, o conhecimento do perfil, do histórico e da adesão do paciente a esse tratamento especializado pode permitir refletir sobre melhorias na assistência e na gestão desse serviço. Novos estudos, como ensaios clínicos, são necessários para avaliar diferentes abordagens terapêuticas com os desfechos adesão e abstinência, por exemplo. Esta pesquisa não teve como objetivo investigar tratamento, e sim caracterizar o usuário, seu perfil, as drogas mais utilizadas, os tratamentos anteriores e sua adesão. Espera-se que este trabalho traga subsídios ao desenvolvimento e ao aprimoramento de políticas públicas de tratamento ao usuário de substância psicoativa, com o intuito de prevenir recaídas e promover programas que auxiliem a qualidade de vida desses usuários e de sua família, criando intervenções rápidas focadas nos fatores de proteção, nas estratégias de enfrentamento e na manutenção da abstinência.

As informações obtidas neste estudo são relevantes e evidenciam a necessidade de novas pesquisas. Dessa forma, elas são necessárias para que a discussão sobre o perfil do usuário brasileiro de substâncias possa traçar um direcionamento de tratamento interdisciplinar que não atue apenas na área da saúde, mas também nos âmbitos político, econômico, social e cultural.

\title{
Comparative study of historical, socio- demographic and adherence characteristics of psychoactive substances users in public outpatient program
}

\begin{abstract}
The use of licit and illicit substances has grown recently in Brazil. It leads to political, economic, social, and cultural problems. Thus, there was also an increase of demand for specialized treatments. So, it is extremely impor-
\end{abstract}


tant to study and categorize the users' profiles to improve the management of the provided service. To define the population served in alcohol and drugs Medical Specialties Clinic treatment program, comparing data from the first year of the service to the data from the second year regarding characteristics in history, socio-demography and patient adherence to treatment. Observational, descriptive, and retrospective study. The sample included 880 patients. Associations between categorical variables by using Fisher's chisquared and exact tests were observed. Profile with predominance of males in both years. In the first year, singles sought treatment, and in the second one, the number of married people increased. Average age was 37, classes $\mathrm{C}$ and D (first year), and $\mathrm{E}$ (second year). Most held previous treatments, with an average of 2.6 treatments. $97.1 \%$ used alcohol, $85.8 \%$ and $68.0 \%$ used tobacco and marijuana. The sample of the first year had a higher percentage of improvement (8.0\% vs. $4.2 \%$ ) and abandonment (39.2\% against $26.5 \%$ ). By categorizing the user, it was evident that their profile fitted what was previously identified by other researchers for Brazilian users (men, from social classes C, D and E, at ages between 30-40 years-old); lawful drugs are the most used ones, which facilitates the consumption. Because drug abuse is a recurring condition, the average of previous treatments is 2.6, which negatively affects adherence. Therefore, it is important to know such data so that treatment management becomes easier, and so that that treatment becomes more effective.

\section{Keywords}

Drug users. Ambulatory care. Substance-related disorders. Health services administration. Medical records.

\section{Estudio comparativo de características históricas, sociodemográficas y de adherencia de usuarios de sustancias psicoactivas en un programa público ambulatorio}

\section{Resumen}

En los últimos años, el consumo de sustancias legales e ilegales en Brasil ha crecido. Esto desencadena problemas políticos, económicos, sociales y culturales. Por lo tanto, hubo un aumento en la demanda de tratamientos especializados. En este sentido, conocer el perfil del usuario y la adherencia al tratamiento es de suma importancia para facilitar la gestión del servicio especializado. Caracterizar la población atendida en el programa de trata- 
miento de alcohol y drogas del Ambulatorio Médico, comparando los datos del primer año de operación del servicio con los del segundo, con respecto a las características históricas, sociodemográficas y de adherencia del paciente al tratamiento. Estudio observacional, descriptivo y retrospectivo. La muestra comprendió 880 pacientes. Se verificaron asociaciones entre variables categóricas utilizando la prueba de chi-cuadrado y la prueba exacta de Fisher. Perfil con predominio de hombres en ambos años. En el primer año, solteros buscaron tratamiento, y en el segundo, el número de personas casadas aumentó. Edad media de 37 años, clase social C y D, y E en el segundo año. La mayoría se sometió a tratamiento previo, con un promedio de 2,6 tratamientos. El 97,1\% consumió alcohol, el 85,8\% de tabaco y el $68,0 \%$ de marijuana. La muestra del primer año mostró un mayor porcentaje de mejoría ( $8,0 \%$ contra $4,2 \%$ ) y abandono $(39,2 \%$ contra $26,5 \%)$. Al caracterizar al usuario, fue posible percibir datos que corroboran lo que otros investigadores habían identificado en usuarios brasileños (hombres, clases C, D y E, grupo de edad entre 30-40 años); las drogas más utilizadas son las lícitas, lo que facilita su uso. Al tratarse de una enfermedad recurrente, la media es de 2,6 tratamientos previos, lo que incide negativamente en la adherencia. Por tanto, es importante conocer estos datos para facilitar la gestión del tratamiento, haciéndolo más eficaz.

\section{Palabras clave}

Consumidores de drogas. Atención ambulatoria. Trastornos relacionados con sustancias. Administración de los servicios de salud. Registros médicos.

\section{REFERÊNCIAS}

ALMEIDA, R. A. et al. Perfil dos usuários de substâncias psicoativas de João Pessoa. Saúde Debate, v. 38, n. 102, p. 526-538, 2014. DOI: 10.5935/0103-1104.20140049

ARAÚJO, R. B. et al. Perfil do usuário de crack e fatores relacionados à criminalidade em unidade de internação para desintoxicação no Hospital Psiquiátrico São Pedro de Porto Alegre (RS). Revista de Psiquiatria do Rio Grande do Sul, v. 30, n. 2, p. 101-108, 2008. DOI: 10.1590/S0101-81082008000300005

ARRUDA, E. F. de et al. Perfil dos usuários de substâncias psicoativas atendidos em um centro de atenção psicossocial do Acre. Enciclopédia Biosfera, v. 16, n. 30, p. 450-469, 2019. DOI: 10.18677/EnciBio_2019B45

ASLANI, P. et al. Adherence: a review of education, research, practice and policy in Spain. Pharmacy Practice, v. 7, n. 3, p. 125-38, 2009. DOI: 10.4321/s188636552009000300001 
BELL, J. et al. Opioid agonist pharmacotherapy in New South Wales from 1985 to 2006: patient characteristics and patterns and predictors of treatment retention. Addiction, v. 104, n. 8, p. 1363-1372, 2009. DOI: 10.1111/j.1360-0443.2009.02633.x

CAPISTRANO, F. C. et al. Perfil sociodemográfico e clínico de dependentes químicos em tratamento: análise de prontuários. Escola Anna Nery, Rio de Janeiro, v. 17, n. 2, p. 234-241, 2013. DOI: 10.1590/S1414-81452013000200005

CHAVES, T. V. et al. Fissura por crack: comportamentos e estratégias de controle de usuários e ex-usuários. Revista de Saúde Pública, v. 45, n. 6, p. 1168-1175, 2011. DOI: 10.1590/S0034-89102011005000066

CHEDA, J. N. D. et al. Impacto do perfil clínico e sociodemográfico na adesão ao tratamento de pacientes de um Centro de Atenção Psicossocial a Usuários de Álcool e Drogas (CAPSad). Jornal Brasileiro de Psiquiatria, v. 59, n. 4, p. 317-321, 2010. DOI: 10.1590/S0047-20852010000400008

CORDEIRO, D. C.; DIEHL, A.; LARANJEIRA, R. R. Organização de serviços de tratamento em dependência química. In: CORDEIRO, D. C.; DIEHL, A.; LARANJEIRA, R. R. (org.). Dependência química: prevenção, tratamento e políticas públicas. Porto Alegre: Artmed, 2019.

CORDEIRO, D. C.; DIEHL, A.; MARI, J. de J. Comorbidades psiquiátricas. In: CORDEIRO, D. C.; DIEHL, A.; LARANJEIRA, R. R. (org.). Dependência química: prevenção, tratamento e políticas públicas. Porto Alegre: Artmed, 2019.

DIEHL, A.; SANTANA, P. R. O. H. Políticas públicas para o controle do álcool, tabaco e outras drogas. In: CORDEIRO, D. C.; DIEHL, A.; LARANJEIRA, R. R. (org.). Dependência química: prevenção, tratamento e políticas públicas. Porto Alegre: Artmed, 2019.

FERREIRA, S. L.; MARINHO, J. A. C.; SILVA, I. F. da. Terapia de rede social e de 12 passos. In: CORDEIRO, D. C.; DIEHL, A.; LARANJEIRA, R. R. (org.). Dependência química: prevenção, tratamento e políticas públicas. Porto Alegre: Artmed, 2019.

FIGLIE, N. B.; LARANJEIRA, R. Gerenciamento de caso aplicado ao tratamento da dependência do álcool. Revista Brasileira de Psiquiatria, São Paulo, v. 26, n. 1, p. 63-67, 2004. DOI: 10.1590/S1516-44462004000500016

GARCIA, M. L. T.; SIQUEIRA, M. M.; SOUZA, R.S. O impacto das faltas às consultas em um programa de dependentes de álcool. Jornal Brasileiro de Psiquiatria, v. 54, n. 2, p. 114-119, abr./jun. 2005. LILACS ID: lil-438302

LARANJEIRA, R. II Levantamento Nacional de álcool e drogas (LENAD). São Paulo: Inpad, Unifesp, 2014.

LARANJEIRA, R.; RIBEIRO, M. (org.). O tratamento do usuário de crack. São Paulo: Casa Leitura Médica, 2010. 
LEITE, S. N.; VASCONCELLOS, M. P. C. Adesão à terapêutica medicamentosa: elementos para a discussão de conceitos e pressupostos adotados na literatura. Ciência e Saúde Coletiva, v. 8, n. 3, p. 775-782, 2003. DOI: 10.1590/S1413-81232003000300011

NAPPO, S. A.; OLIVEIRA, L. G de. Crack na cidade de São Paulo: acessibilidade, estratégias de mercado e formas de uso. Revista Psiquiatria Clínica, v. 35, n. 6, p. 212-218, 2008. DOI: 10.1590/S0101-60832008000600002

PECHANSKY, F.; SZOBOT, C. M.; SCIVOLETTO, S. Uso de álcool entre adolescentes: conceitos, características epidemiológicas e fatores etiopatogênicos. Revista Brasileira de Psiquiatria, v. 26, supl. 1, p. 14-17, 2004. DOI: 10.1590/S1516-44462004000500005 RIBEIRO, D. R.; CARVALHO, D. S. O padrão de uso de drogas por grupos em diferentes fases de tratamento nos Centros de Atenção Psicossocial Álcool e Drogas (CAPS-AD). Jornal Brasileiro de Psiquiatria, v. 64, n. 3, p. 221-229, 2015. DOI: 10.1590/00472085000000082

SILVA, C. J. da. Dependência química no Brasil e o papel das organizações sociais na gestão dos serviços de tratamento: estudo do caso. 2013. Monografia (MBA em Gestão de Saúde) - Instituto de Ensino e Pesquisa, São Paulo, 2013.

SILVA, M. L.; GUIMARÃES, C. F; SALLES. D. B. Fatores de risco e proteção à recaída na percepção de usuários de substâncias psicoativas. Revista Rene, v. 15, n. 6, p. 1007-1015, 2014. DOI: $10.15253 / 2175-6783.2014000600014$

ZANELATTO, N. Tratamento ambulatorial. In: CORDEIRO, D. C.; DIEHL, A.; LARANJEIRA, R. R. (org.). Dependência química: prevenção, tratamento e políticas públicas. Porto Alegre: Artmed, 2019. 